\title{
Research Paper \\ Investigating the Relationship Between Corona Anxiety and Nursing Care Behaviors Working in Corona's Referral Hospitals
}

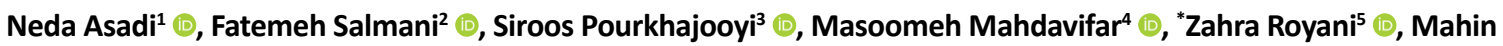
Salmani ${ }^{6}$ (1)

1. Nursing Research Center, Kerman University of Medical Sciences, Kerman, Iran.

2. Nursing \& Midwifery Sciences Development Research Center, Najafabad Branch, Islamic Azad University, Najafabad, Iran.

3. Department of Health Economics, Policy and Management, Faculty of Management and Medical Information Sciences, Kerman University of Medical Sciences, Kerman, Iran.

4. Department of Nursing, Faculty of Nursing \& Midwifery, Hormozgan University of Medical Science, Bandar Abbas, Iran.

5. Department of Nursing, Faculty of Paramedical Sciences, Golestan University of Medical Sciences, Gorgan, Iran.

6. Department of Mathematics and Statistics, Faculty of Science, University of New Brunswick, Fredericton, Canada.

\begin{tabular}{|c|c|}
\hline $\begin{array}{l}\text { Use your device to scan } \\
\text { and read the article online }\end{array}$ & dettion Asadi N, Salmani F, Pourkhajooyi S, Mahdavifar M, Rooyani Z, Mahin S. Investigating the Relationship Between \\
\hline 口ifitit口 & Corona Anxiety and Nursing Care Behaviors Working in Coronary Referral Hospitals. Iranian Journal of Psychiatry and Clinical \\
\hline 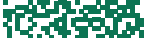 & Psychology. 2020; 26(3):306-319. http://dx.doi.org/10.32598/ijpcp.26.3476.1 \\
\hline - $45 \mathrm{~s}$ & doijhttp://dx.doi.org/10.32598/ijpcp.26.3476.1 \\
\hline
\end{tabular}

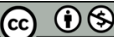

Received: 19 Jun 2020

Accepted: 16 Sep 2020

Available Online: 01 Dec 2020

Key words:

Caring, Behavior, Nurs-

es, Anxiety, COVID-19

\section{A B STRACT}

Objectives The prevalence of Coronavirus and its health-related psychosocial consequences is one of the most important human social events of the $21^{\text {st }}$ century. Nurses, due to close contact with patients, are vulnerable to be infected with Covid-19. Therefore, they face severe psychological consequences. This study aimed to determine the relationship between Corona's anxiety and nursing care behaviors in working in Corona referral hospitals in Kerman in 2020.

Methods The present study is cross-sectional descriptive-correlational research. Sampling was performed by the census method. A total of 166 nurses entered the study. In the present study, three demographic questionnaires, the Corona Disease Anxiety Scale (CDAS) and Caring Behaviors Inventory (CBI) were used. The analysis was done using Descriptive and Inferential statistics SPSS V. 18 software Results The overall score of Corona anxiety among the nurses was $21.39 \pm 9.8$, and the overall score of the nursing behavior of the studied nurses was $109.7 \pm 4.2$ with a range of 94 to 118 . Spearman's correlation coefficient showed that there was no significant relationship between corona anxiety and caring behaviors. Conclusion The present study showed that nurses working in corona wards suffer from moderate anxiety, and the level of caring behaviors provided by nurses was optimal. According to the current study findings, it is suggested that during the outbreak of emerging and epidemic diseases, to reduce nursing staff's anxiety, coping strategies and resilience skills, and problem-solving, managers should pay more attention.

\section{Extended Abstract

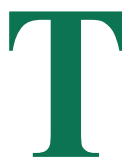 \\ 1. Introduction \\ he prevalence of Coronavirus and its health-related social consequences is one \\ of the most important human social events of the $21^{\text {st }}$ cen- tury [4]. What distinguishes this spread is people's irra- tional behavior due to fear of exposure to the disease [5]. One of the most important consequences of the outbreak of the Coronavirus is social anxiety [6]. Hospital healthcare personnel are always at the forefront of specific epidemics}

* Corresponding Author:

Zahra Royani, MSc.

Address:Department of Nursing, Faculty of Paramedical Sciences, Golestan University of Medical Sciences, Gorgan, Iran

Tel: +98(173) 2436102

E-mail: z.royani@goums.ac.ir 
and risk their lives to perform their duties [9]. Meanwhile, nurses are at the forefront of fighting infectious diseases in close contact with COVID-19; thus, they are vulnerable to infection [34].

In addition to physical stress, they also face tremendous pressure. Because of the nature of the nursing job, it leads to long-term contact with infected patients, and in these critical situations, they provide care as the most basic nursing job [11]. The process of providing nursing care is influenced by nurses' working environment [16]. There are still concerns about nurses' capacity in the field of care, given the COVID 19 pandemic in the world [20]. Therefore, the present study aimed to investigate the relationship between Corona's anxiety and nursing care behaviors in nurses working in Corona's Referral Hospitals in Kerman City in 2020.

\section{Methods}

The present study is a cross-sectional descriptive-correlational study conducted in 2020 to determine the relationship between care behaviors and Corona's anxiety in nurses working in Corona's referral hospitals in Kerman city. Due to the limited research population, sampling was performed by the census method.

A total of 166 nurses entered the study. Due to the conditions prevailing in the research environment and the limited presence in medical centers, the research was conducted electronically. In other words, the electronic form of the questionnaires, along with the conscious consent form for participating in the study, was designed and uploaded using the online Porsline software. Three questionnaires used demographic Corona Disease Anxiety Scale (CDAS) and Caring Behaviors Inventory (CBI) in the present study.

The demographic and contextual information questionnaire included age, gender, workplace, work experience, education level, and more. CDAS has been prepared and validated to measure anxiety caused by the prevalence of Coronavirus in Iran [21]. The CBI, containing 25 items, was used. Its validity and reliability have been confirmed by Watson et al. (1997) [10], which mainly covers the professional psychological and technical aspects of care. In Iran, the questionnaire's validity has been confirmed through formal and content validity and instrumentation reliability. Cronbach's alpha coefficient was reported to be $\alpha=0.93$ [11]. The analysis was performed in SPSS v. 18 software using descriptive and empirical statistics by Spearman correlation coefficient tests and Mann-Whitney U and Kruskal-Wallis H tests.

\section{Results}

A total of 166 nurses participated in this study. Corona's overall score in the nurses studied was $21.39 \pm 9.8$, and the overall score of the nurses in the survey was 109.7 \pm 4.2 ranged 94-118. Spearman's correlation coefficient showed no significant relationship between care behaviors and Corona's anxiety $(\mathrm{P}>0.05)$. It should be noted that the number of items in each subscale was different. Therefore, to make the comparison possible, the scores obtained from each subscale and the number of things related to it divided total care behaviors.

The average scores that can be obtained in each case are between 1-5 scores. The highest mean was related to the dimension of professional behaviors with a mean of $4.81 \pm 0.48$, and the lowest standard was associated with the measurement of inappropriate behavior of $1.25 \pm 0.40$. Mann-Whitney $U$ test showed that Coronass anxiety and care behaviors did not significantly differ between the sexes. The Kruskal-Wallis $\mathrm{H}$ test showed that Corona>s anxiety and care behaviors did not differ significantly between people with different education and employment in other work areas (Table $1 \& 2$ ). Spearman $>$ s correlation coefficient showed that there was no significant relationship between corona anxiety and caring behavior. In studying psychological and physical dimensions of anxiety, no meaningful relationship was found with subgroups of care behaviors (unnecessary actions, professional behaviors, psychosocial behaviors, inappropriate behaviors, physical-technical behaviors) (Table 3).

\section{Discussion and Conclusions}

The present study showed that COVID-19 is a source of anxiety for nurses directly exposed to it. Basically, COVID-19 is a source of great anxiety for all people and different social groups. Different people experience different psychological levels of the crisis, especially people like nurses at the center of the crisis [11]. Another finding of this study was that in general, the mean score of nursing behavior in nurses was high, indicating that nursing behavior was very desirable, and the findings of this study showed no significant relationship between Corona's anxiety and nursing care behaviors. Despite having anxiety, nurses tried to play patient care behaviors as the most fundamental role of nursing. Overall, the medical staff has shown great compassion in the face of the COVID-19 pandemic globally, while it was beyond their official duty [35]. These results also confirm that nurses in our country, as one of the largest medical staff groups, were no exception to this rule. However, it seems necessary to provide psychological support to nurses and provide training to deal with corona anxiety in the current crisis. 
Table 1. Demographic characteristics of research samples

\begin{tabular}{ccc}
\hline & Variables & No. (\%) \\
\hline Sex & Men & $71(8.42)$ \\
& Female & $95(2.57)$ \\
\hline Education & BSc. & $142(5.85)$ \\
& MSc. & $20(12)$ \\
Unite & PhD. & $4(5.2)$ \\
& Emergency & $40(1.24)$ \\
\hline Age & General & $30(18)$ \\
& Intensive care unite & $51(7.30)$ \\
\hline CoviD-19 & & $45(2.27)$ \\
\hline
\end{tabular}

Iranian Journal of
PSYCHIATRY AND CUNICAL PSYCHOLOGY

Table 2. Determination and comparison of the mean score of care behavior and Corona's anxiety based

\begin{tabular}{|c|c|c|c|}
\hline \multirow{2}{*}{\multicolumn{2}{|c|}{ Variables }} & \multicolumn{2}{|c|}{ Mean \pm SD } \\
\hline & & Care Behavior & COVID Anxiety \\
\hline \multirow{3}{*}{ Sex } & Female & $116 \pm 4.6$ & $20 \pm 9.1$ \\
\hline & Male & $117 \pm 8.3$ & $22 \pm 10.4$ \\
\hline & - & $\mathrm{P}=0.39$ & $P=0.126$ \\
\hline \multirow{4}{*}{ Education level } & Bachelor & $116.5 \pm 4.2$ & $22.7 \pm 10.8$ \\
\hline & Master of Science & $116.9 \pm 3.9$ & $18.2 \pm 6.6$ \\
\hline & PhD. & $117.5 \pm 5.5$ & $22.2 \pm 9.9$ \\
\hline & - & $P=0.63$ & $P=0.110$ \\
\hline \multirow{5}{*}{ Unite } & Emergency & $117.1 \pm 9.3$ & $22.1 \pm 10.6$ \\
\hline & General & $117.4 \pm 7.3$ & $19.9 \pm 9.4$ \\
\hline & Critical care & $115.4 \pm 9.4$ & $21.1 \pm 8.8$ \\
\hline & Covid & $118.2 \pm 2.4$ & $36.1 \pm 6.2$ \\
\hline & - & $\mathrm{P}=0.17$ & $\mathrm{P}=0.32$ \\
\hline \multirow{2}{*}{\multicolumn{2}{|c|}{ Age }} & $r=0.029$ & $r=0.11$ \\
\hline & & $P=0.17$ & $\mathrm{P}=0.123$ \\
\hline
\end{tabular}


Table 3. The relationship between care behavior and Corona's anxiety in nurses

\begin{tabular}{|c|c|c|c|c|c|c|}
\hline Variable & $\begin{array}{c}\text { Physical_technical } \\
\text { Behaviors }\end{array}$ & $\begin{array}{l}\text { Improper } \\
\text { Behavior }\end{array}$ & $\begin{array}{l}\text { Psychosocial } \\
\text { Behaviors }\end{array}$ & $\begin{array}{l}\text { Profes- } \\
\text { sional } \\
\text { Behaviors }\end{array}$ & $\begin{array}{l}\text { Unnecessary } \\
\text { Behaviors }\end{array}$ & $\begin{array}{c}\text { Total Score } \\
\text { of Caring } \\
\text { Behavior }\end{array}$ \\
\hline \multirow{2}{*}{ Psychological symptoms of anxiety } & $r=0.03$ & $r=0.06$ & $r=0.001$ & $r=0.05$ & $r=0.11$ & $r=0.03$ \\
\hline & $P=0.66$ & $P=0.43$ & $P=0.98$ & $P=0.47$ & $P=0.14$ & $P=0.7$ \\
\hline \multirow{2}{*}{ Physical symptoms of anxiety } & $r=-0.04$ & $r=0.11$ & $r=-0.01$ & $r=0.000$ & $r=0.06$ & $r=-0.01$ \\
\hline & $P=0.59$ & $P=0.14$ & $P=0.81$ & $P=0.98$ & $P=0.43$ & $P=0.82$ \\
\hline \multirow{2}{*}{ Total score of anxiety } & $r=0.04$ & $r=0.09$ & $r=0.03$ & $r=0.01$ & $r=0.04$ & $r=-0.02$ \\
\hline & $P=0.61$ & $P=0.22$ & $\mathrm{P}=0.7$ & $P=0.83$ & $P=0.6$ & $P=0.7$ \\
\hline
\end{tabular}

Iranian Journal of
PSYCHIATRY AND CUINICAL PSYCHOLOG

\section{Ethical Considerations}

Compliance with ethical guidelines

All ethical principles are observed in this article. Participants were allowed to leave the study if they were reluctant to cooperate in the investigation and assured that their information would remain confidential. This article has received the Research Ethics Committee's approval steps from Kerman University of Medical Sciences with the identification number 1399.181.IR.KMU.REC.

\section{Funding}

This research did not receive any specific grant from funding agencies in the public, commercial, or not-forprofit sectors.

\section{Authors' contributions}

All authors contributed to designing, running, and writing all parts of the research.

\section{Conflicts of interest}

The authors declared no conflict of interest.

\section{Acknowledgements}

The authors would like to thank the Vice-Chancellor for Research and Technology of Kerman University of Medical Sciences, the nursing staff of Afzalipour Hospital and the Payambar Azam Hospital, and all contributors. 
This Page Intentionally Left Blank 


\title{
همبستكى بين اضطراب كرونا و رفتارهاى مراقبتى يرستاران شاغل در بيمارستانهاى ارجاعى كرونا
}

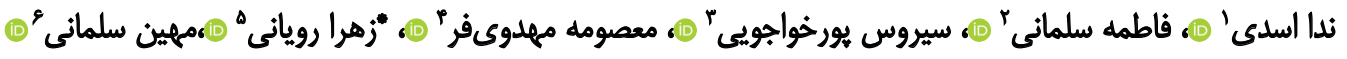 \\ 1. ا.مركز تحقيقات يرستارى، دانشكاه علوميزشكى كرمان، كرمان، ايران.

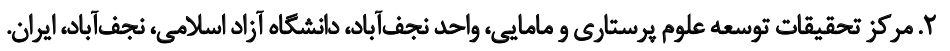

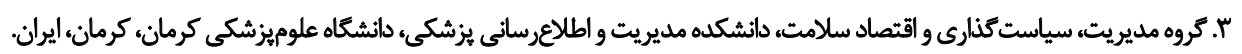

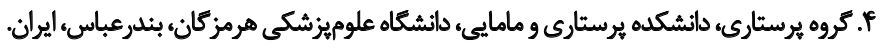

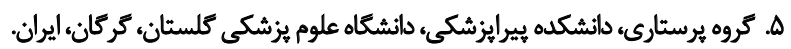

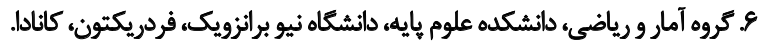

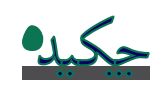

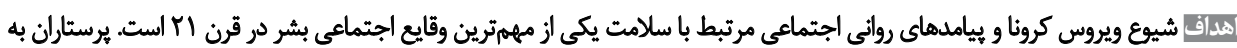

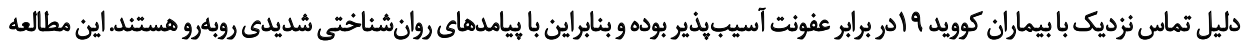

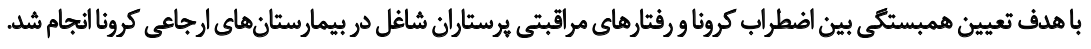

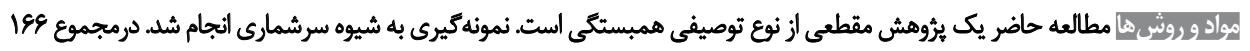

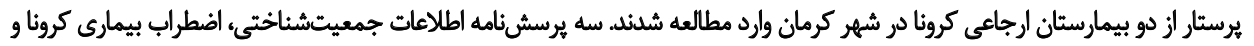

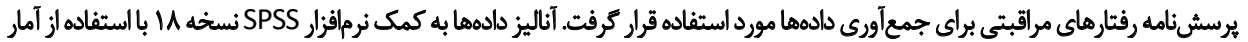
توصيفى و استنباطى انجام شد.

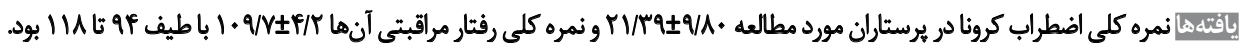

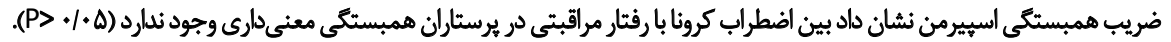

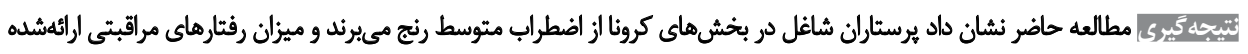

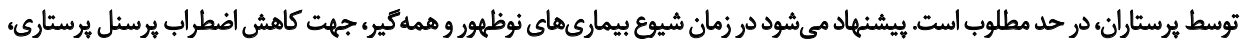

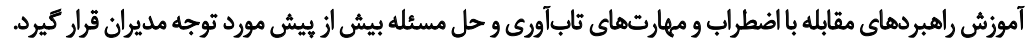

تاريخ دريافت: +r خرداد

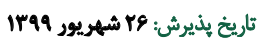

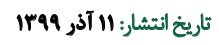

كليدوازهها:

مراقبت، رفتار، يرستاران،

اضطراب، كوويد 19 19تارن

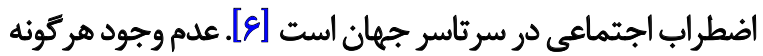

dale

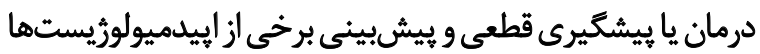

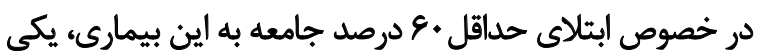

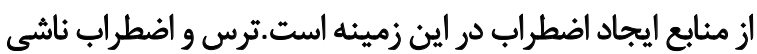

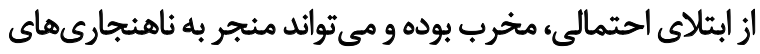

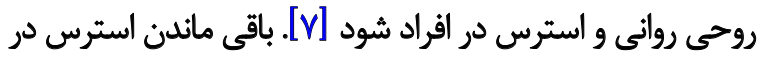

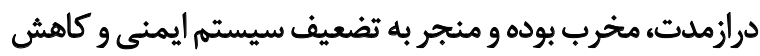

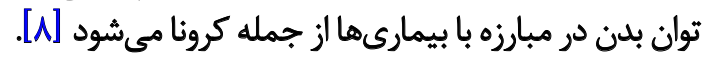

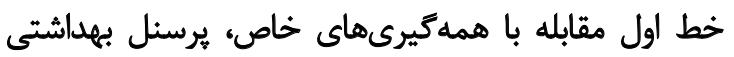

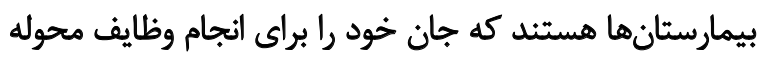

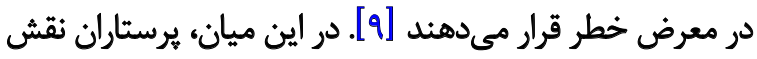

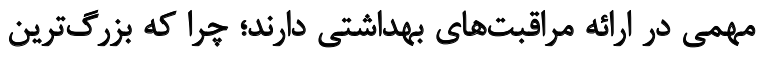

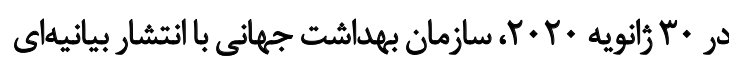

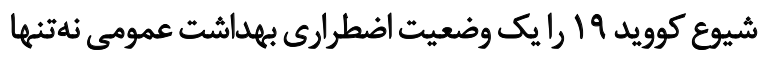

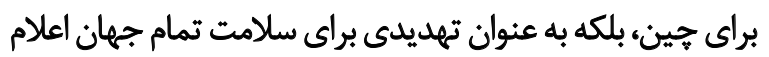

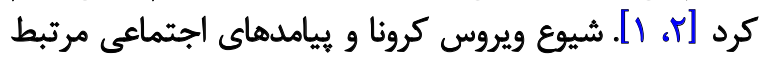

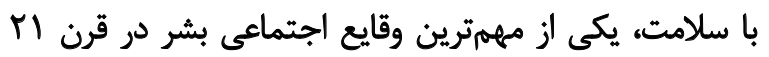

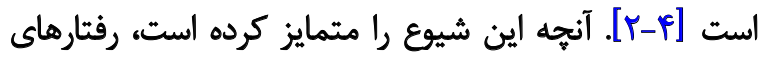

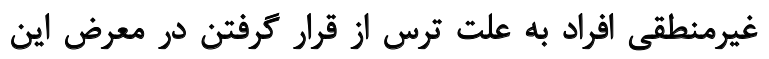

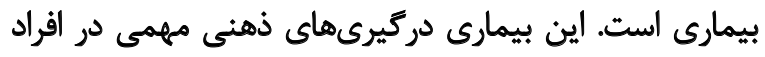

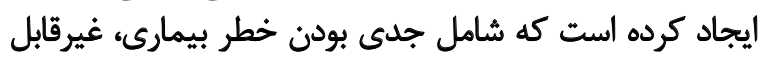

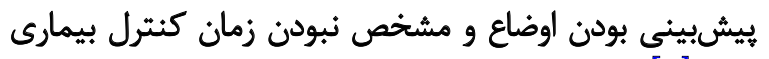

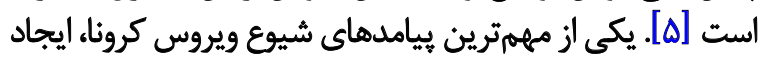

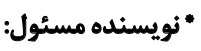

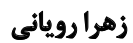

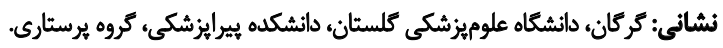

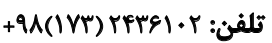
يست الكترونيكي: z.royani@goums.ac.ir 
نيروى انسانى فعال سازمانهاى مراقبتى بهداشتى محسوب

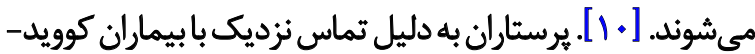
مطالعه حاضر، يروهشى مقطعى از نوع توصيفى همبستيكى

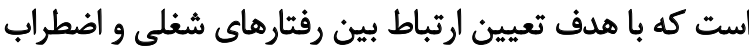

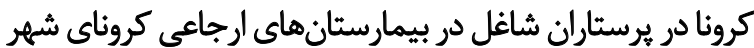

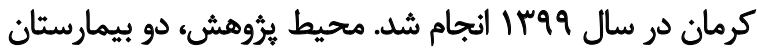

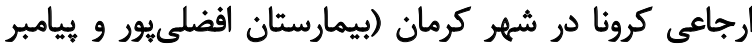

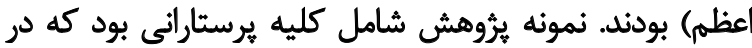

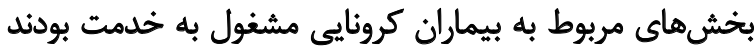

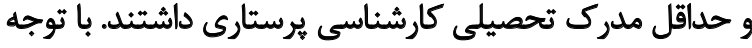

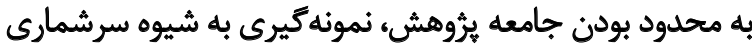

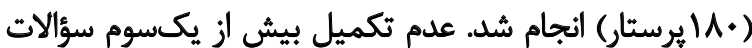

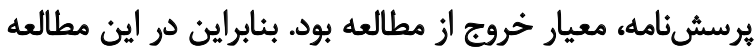

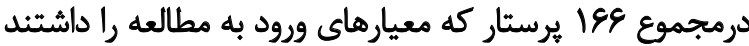

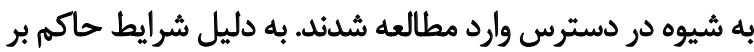

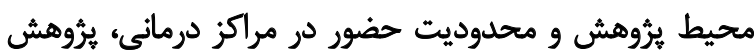

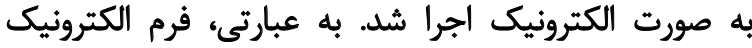

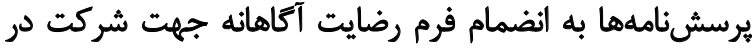

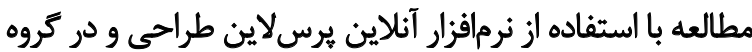

$$
\text { واتساب يرستاران باركذارى شد. }
$$

در مطالعه حاضر از سه يرسشنامه اطلاعات جمعيتشناختئي

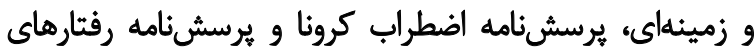

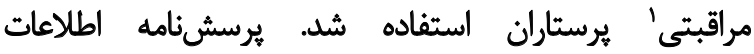

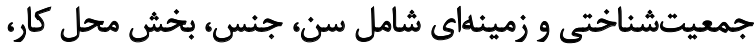

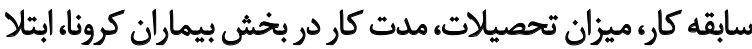

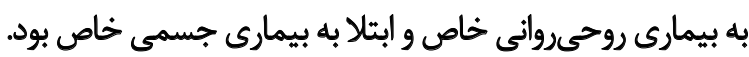
يرسشنامه اضطراب بيمارى كروناّ جهت سنجش اضطراب

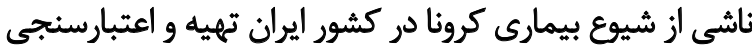

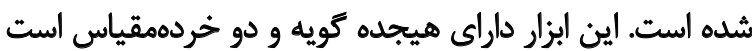

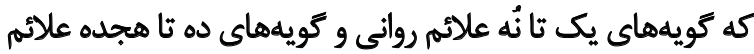

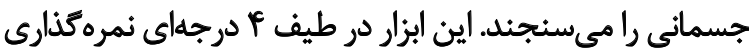

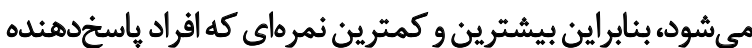

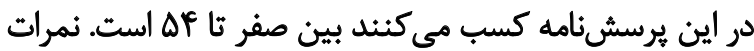

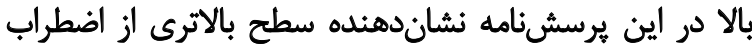

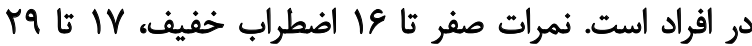

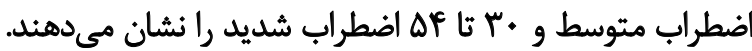

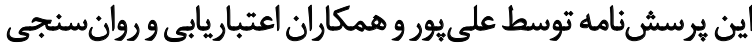

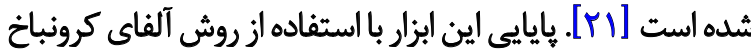

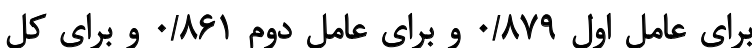
يرسشنامه 9191 • ب به دست آمده است.

يرسشنامه رفتارهاى مراقبتى شامل هY گ گويه است كه عمدتاً

1. Caring Behaviors Inventory (CBI)

2. Corona Disease Anxiety Scale (CDAS)

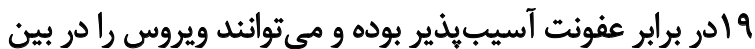

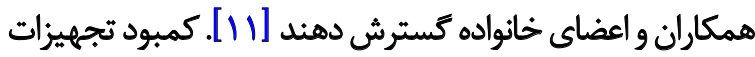

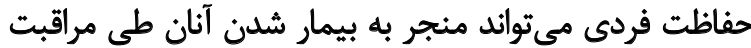

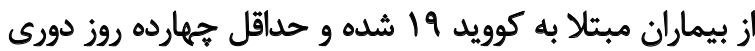

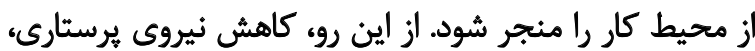

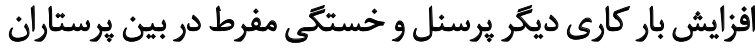

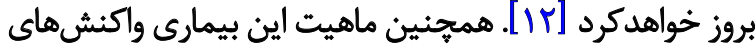

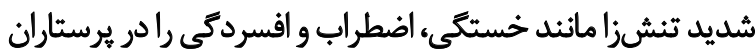

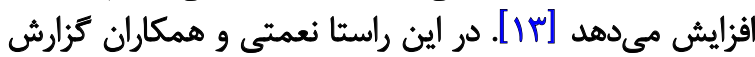

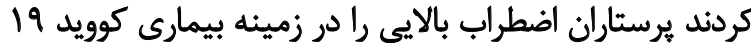

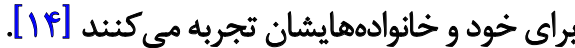

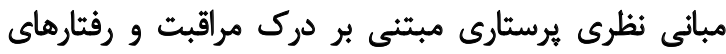

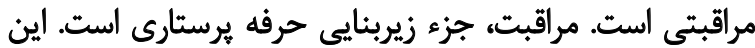

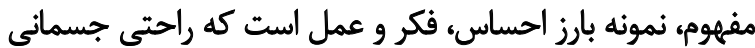

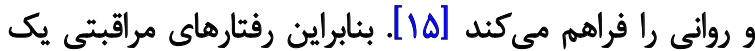

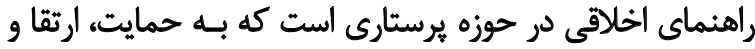

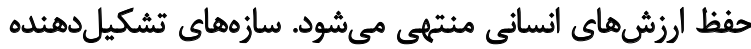

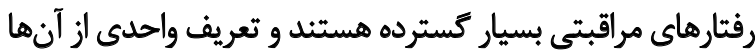

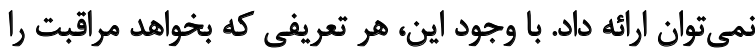

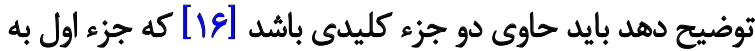

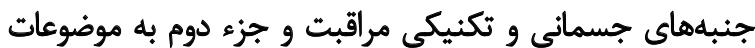

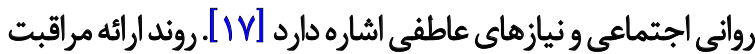

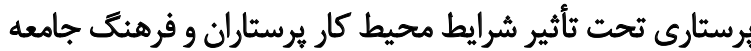

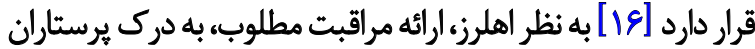

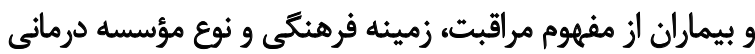

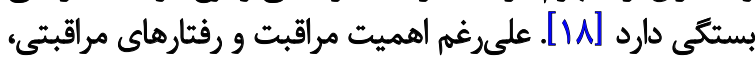

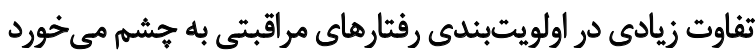

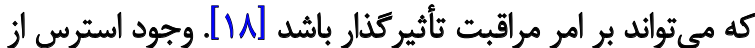

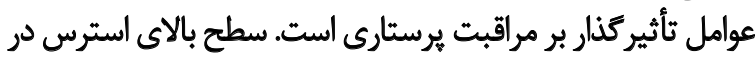

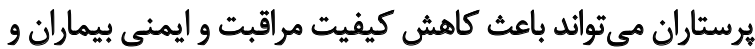

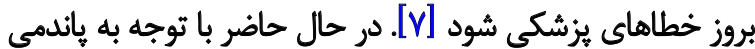

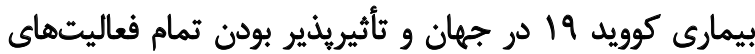

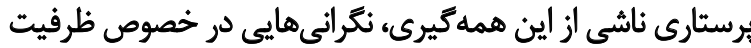

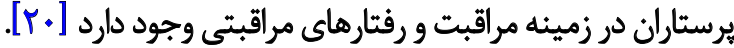

جستوجوى يِيشرفته در مطالعات موجود، حاكى از آن است كه

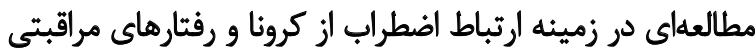

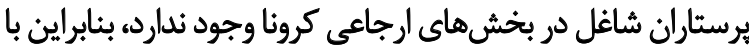

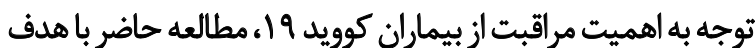

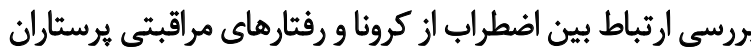

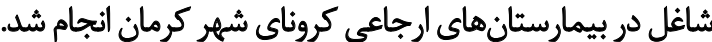


جدول ا. فراوائى مشخصات جمعيتشناختى نمونهماي يثوهش

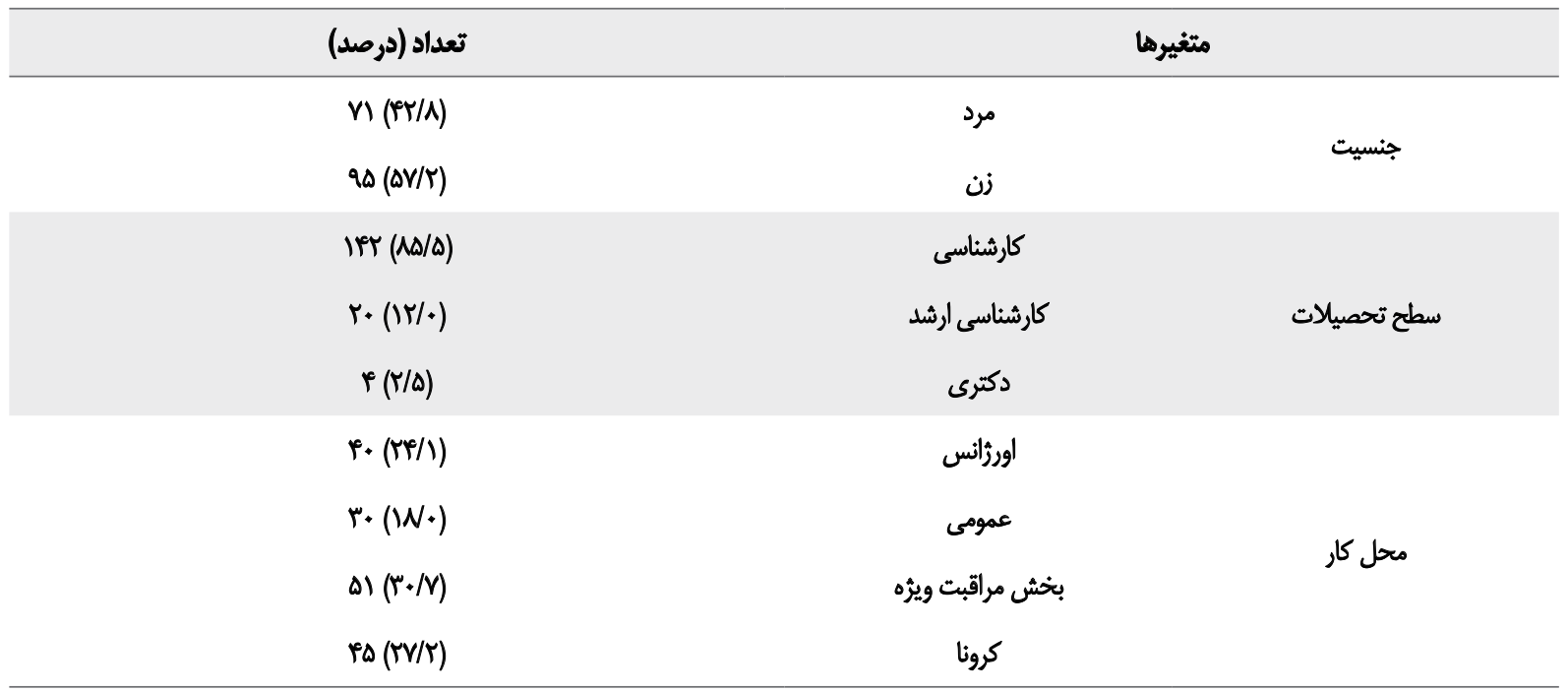

كه تعداد كويههاي هر زيرمقياس متفاوت بود و به منظور ايجاد

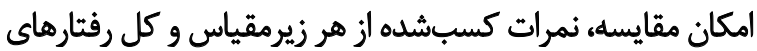

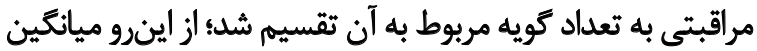

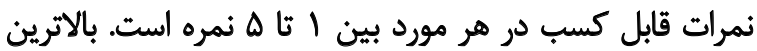

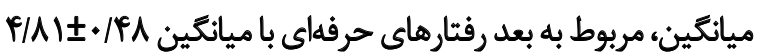

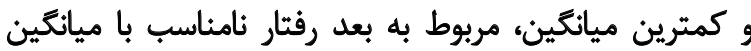

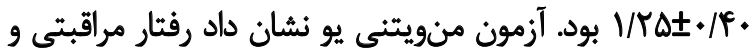

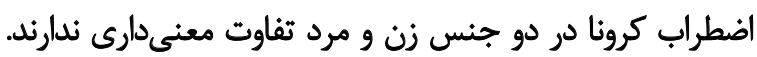

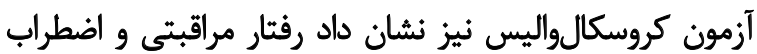

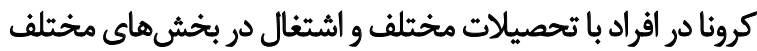

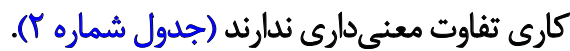

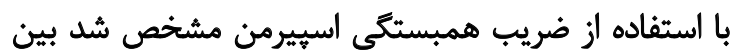

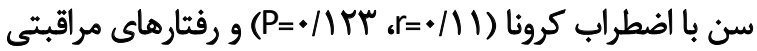

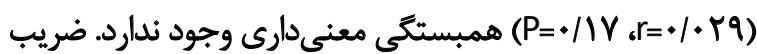

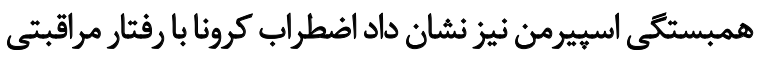

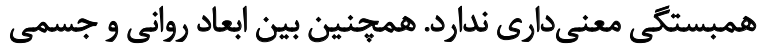

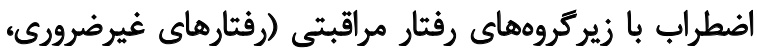

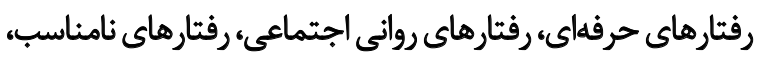

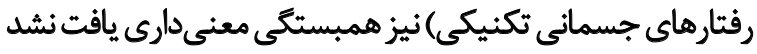

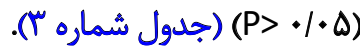

ثs

نتايج مطالعه حاضر نشان داد بين اضطراب كرونا و رفتارهاى

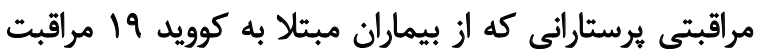

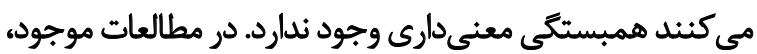

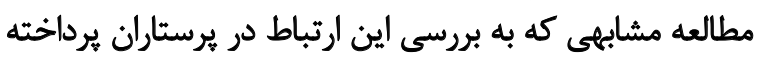

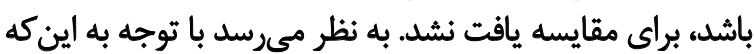

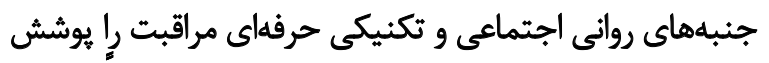

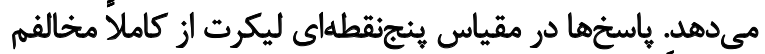

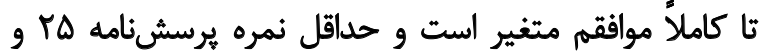

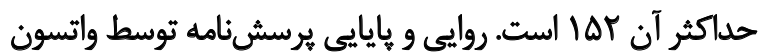

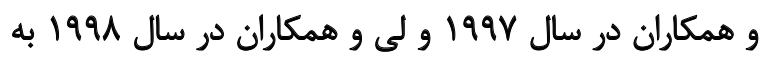

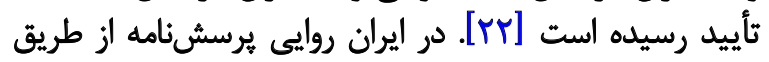

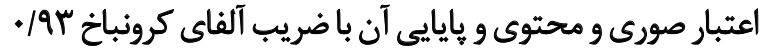

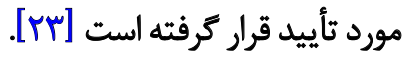

به منظور تجزيه و تحليل دادهها از روش هاى آمارى توصيفى

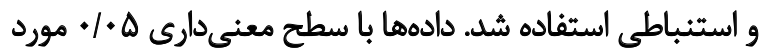

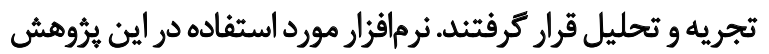

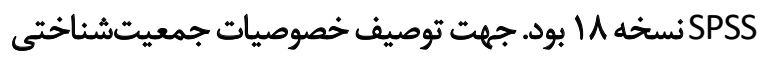

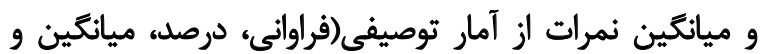

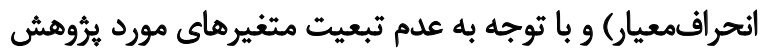

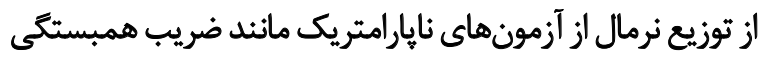

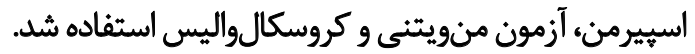

Bdịt

199 يرستار در اين مطالعه شركت كردند. QVVT درصد نمونهها

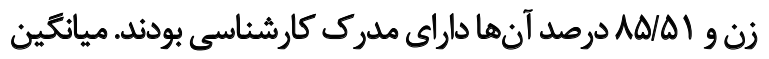

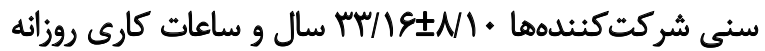

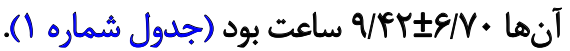

نتايج مطالعه نشان داد نمره كلى اضطراب كرونادر يرستاران مورد

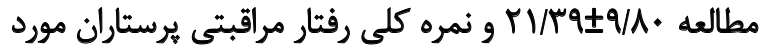

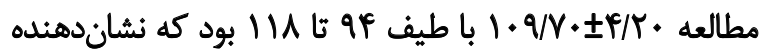
سطح اضطراب متوسط در يرستاران و سطح مطلوب رفئ رفتارهاي

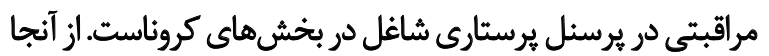


جدول ז. مقايسه ميانكين رفتار مراقبتى و اضطراب كرونا بر اساس مشخصات جمعيتشناختى يرستاران شاغل در سانتر كرونا شهر كرمان سال 9ج9

\begin{tabular}{|c|c|c|c|}
\hline \multicolumn{2}{|c|}{ ميانكين +انحرافمعيار } & \multirow{2}{*}{\multicolumn{2}{|c|}{ مثغيرها }} \\
\hline اضطراب كرونا & رغتار هراقبتى & & \\
\hline$r \cdot 1 \cdot \pm q / 1$ & $\mid 18 / \cdot \pm \varphi / 8$ & ن & \\
\hline$M T / \cdot \pm 1 \cdot / F$ & $\mid I V / \cdot \pm r / A$ & مرد & جنس \\
\hline ./NFE &.$/ 49$ & \multicolumn{2}{|c|}{ سطح معنى دارى } \\
\hline$M r(V \pm 1 \cdot / \mathrm{A}$ & $118 / \Delta \pm f / r$ & كارشناسي & \\
\hline WrIE/E & $118 / 9 \pm r / 9$ & كارشناسي ارششد & سطح تحصيلات \\
\hline$r r / r \pm 9 / q$ & $\| V / \Delta \pm \Delta / \Delta$ & دكترى & \\
\hline.$/ 1$ &. & \multicolumn{2}{|c|}{ سطح معنى دارى } \\
\hline$T T / \Lambda \pm 1 \cdot / 8$ & ||$v / \pm \pm r / q$ & اورخأس & \multirow{4}{*}{ خضش محل خلمث } \\
\hline $19 / 9 \pm 9 / 4$ & $\mid I V / f \pm r / V$ & عمومى & \\
\hline$r / / \pm N A$ & $11 \Delta / f \pm+/ 9$ & ويره & \\
\hline$r g / 1 \pm g / r$ & WWrEt/T & 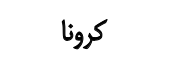 & \\
\hline.$/ T r$ &.$/ \mathrm{V}$ & \multicolumn{2}{|c|}{ سطح معنى دارى } \\
\hline
\end{tabular}

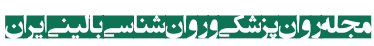

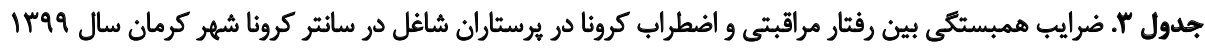

\begin{tabular}{|c|c|c|c|c|c|c|}
\hline نمره كل رقتار مراقبتى & 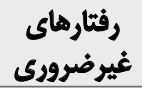 & رفتارهاى حرفهاى & رفتّارهاي روانى - إجتماعى & رفتار هاى نامناسب & رفتار رهاي جسمائى & مثغيرها \\
\hline$\%$ &.$M$ & $\%$ & $.1 .+1$ & .1 .8 & 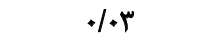 & علائم رواثى اضطراب \\
\hline.$- / .1$ & .1 .8 & $.1 \ldots 1$ & -.1 .1 & .111 &.$- / .8$ & علاثتم جسمانى اضطراب \\
\hline$-* / * r$ & $+1+f$ & .1 .1 & $.1 . \mathrm{T}$ & .1 .9 & $\because * f$ & نمره كل اضطراب \\
\hline
\end{tabular}

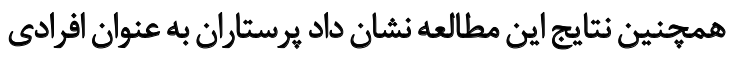

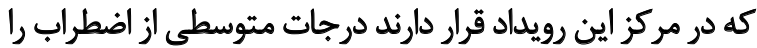

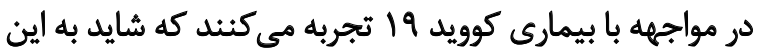

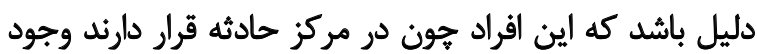

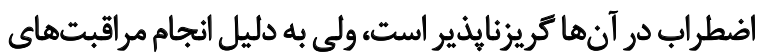

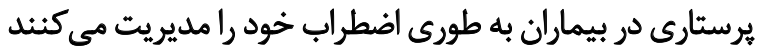

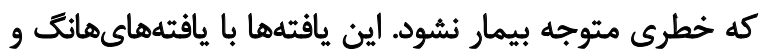

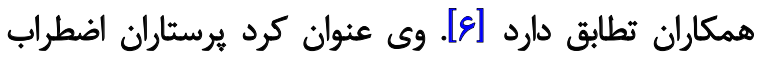

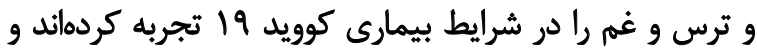

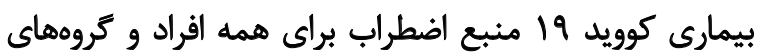

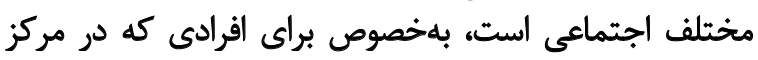
حادثه قرار دارند [11]]
رتباط معنى دارى بين اضطراب كرونا و رفتارهاى مراقبتى وجود نداشت مىتوان كفت كه عدم وجود اين ارتباط بيانكر آن است است كه يرستاران درجات متوسطى از اضطراب رات تجربه مئ مئ كنيند كه

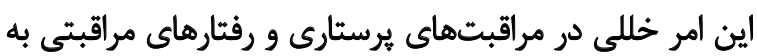

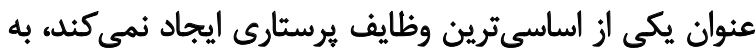

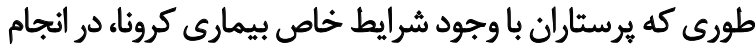

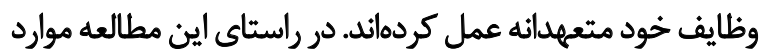

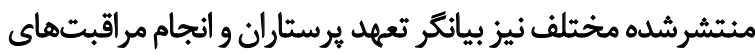
مطلوب در شرايط اضطرابزاي كرونا در كشورهاي جينين، ايتاليا

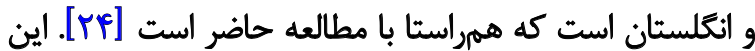

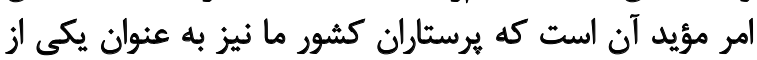

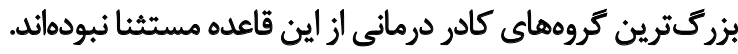


بحرانى زمان كرونا باشد. همجنين با وجود اين كه سطح اضطراب

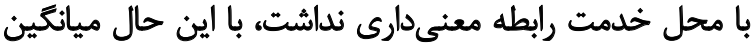

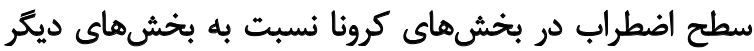

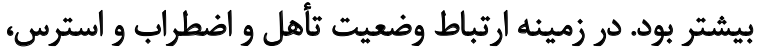

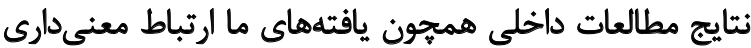

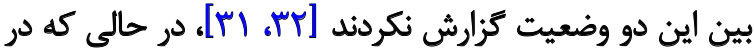

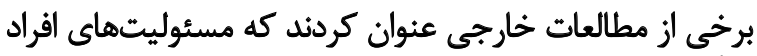

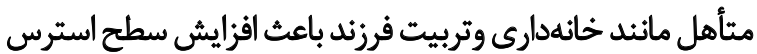

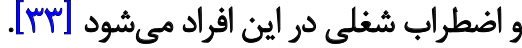

\section{نتيجليَّيرى}

مطالعه حاضر نشان داد برستاران سطح متوسطى از اضطراب

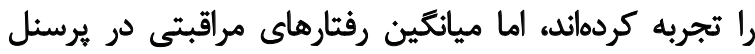

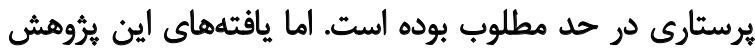

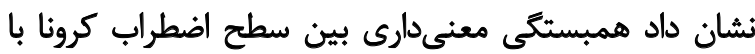

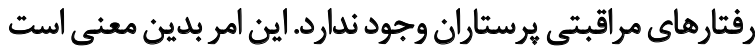

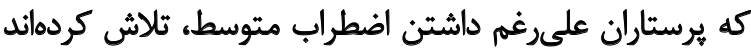

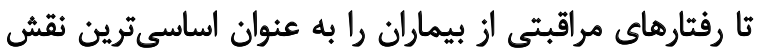

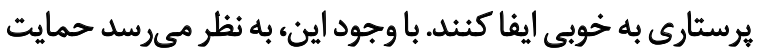

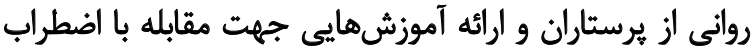
كرونا در شرايط بحران كنوني ضرورى است.

از مهمترين محدوديتهاى مطالعه، وجود تفاوتهاى فردى

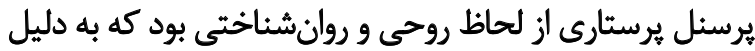

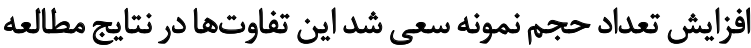

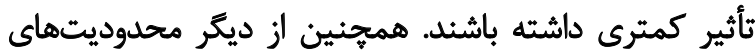

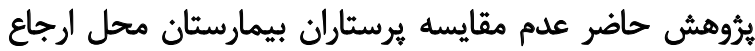

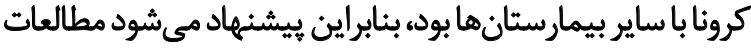

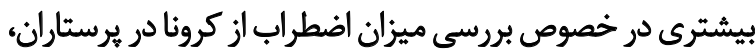

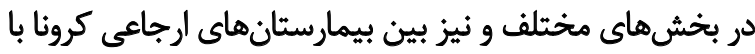
ساير بيمارستانها به صورت مقايسهاي انجام شود.

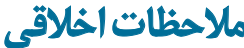

همه اصول اخلاقى در اين مقاله رعايت شده است؛

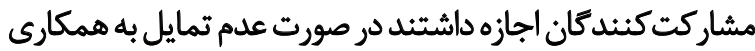

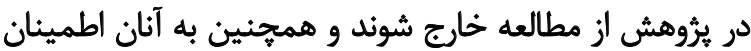

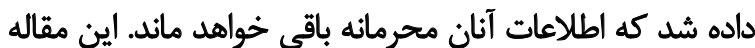

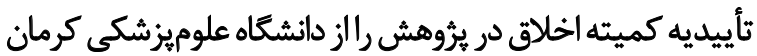
بـا شناسه 1399.181.IR.KMU.REC دريافت كرده است.

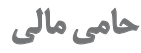

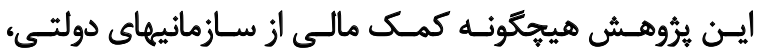

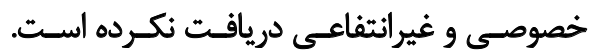

در مطالعه ديكرى كه بإيا و همكاران با عنوان فراوانى اضطراب

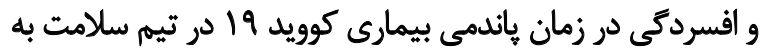

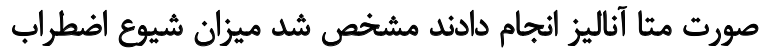

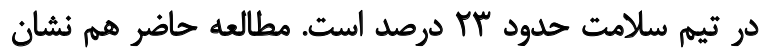

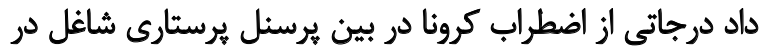

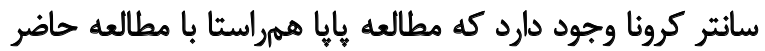

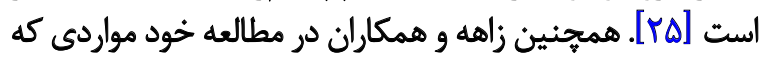

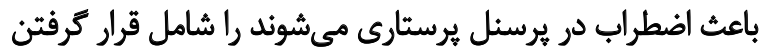

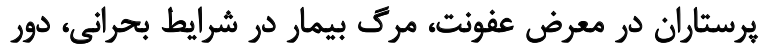

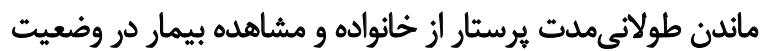

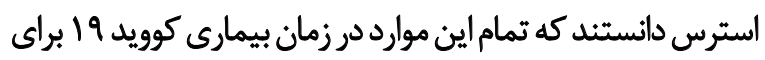

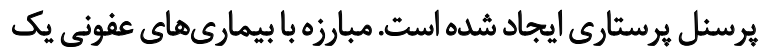

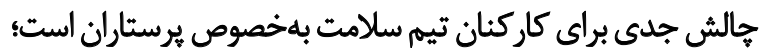

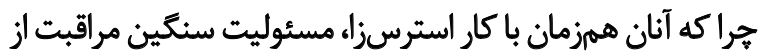

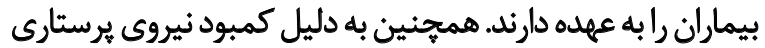

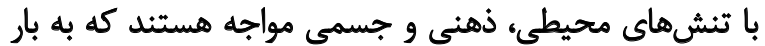

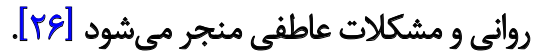

از ديكر يافتههاى اين مطالعه بالا بودن ميانكين نمره رفتار

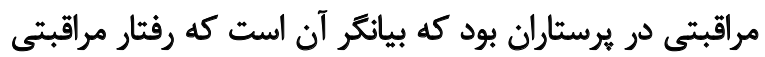

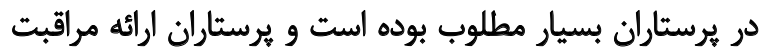

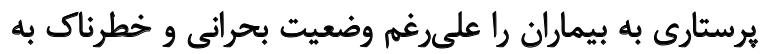

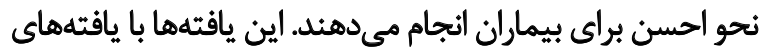

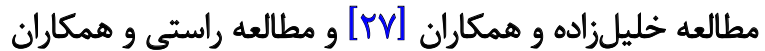

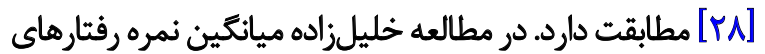

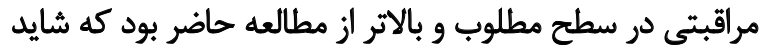

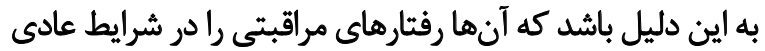

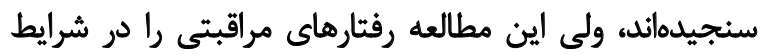

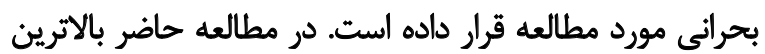

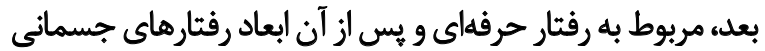

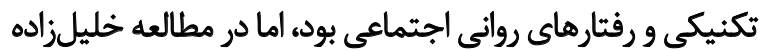

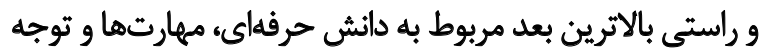

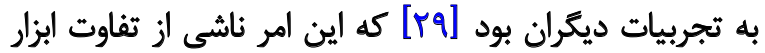

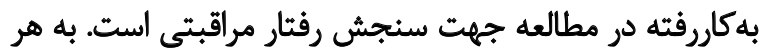

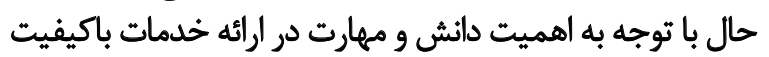

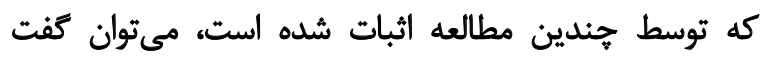

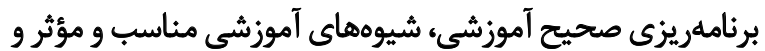

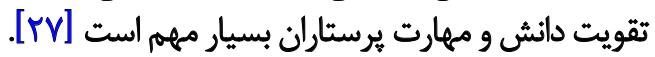
در بررسى رابطه متغيرهاى جمعيتشناختى(سن، جنس،

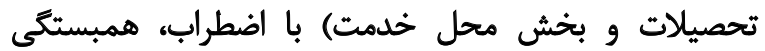

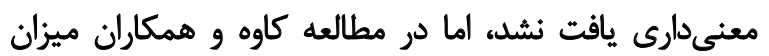

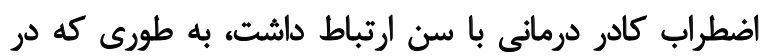

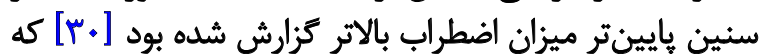

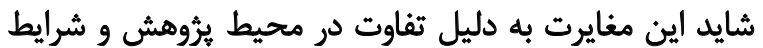




$$
\text { مشار كت نويسيندكُان }
$$

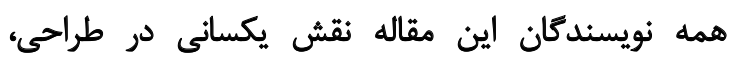

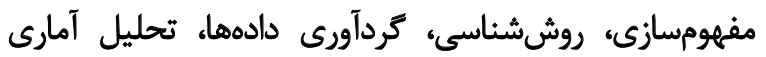

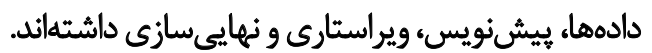

$$
\text { ế }
$$

طبق نظر نويسندكان، اين مطالعه هيج تونه تضاد منافعى

$$
\text { نداشته است. }
$$

$$
\text { تشكر و قدروانى }
$$

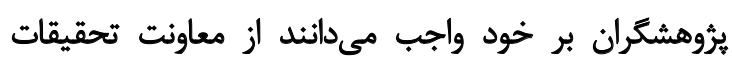

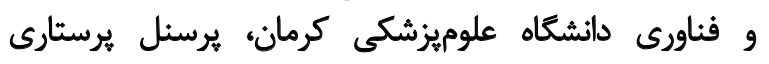

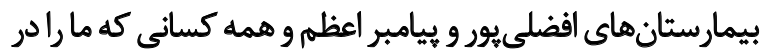
انجام اين يثوهش يارى كردند، تشكر و قدردانى كنند. 


\section{Reference}

[1] Surveillances V. The epidemiological characteristics of an out break of 2019 novel Coronavirus Diseases (COVID-19)China.China CDC Weekly. 2020; 2(8):113-22. [DOI:10.46234/ ccdcw2020.032]

[2] Chan JF-W, Yuan S, Kok KH, To KKW, Chu H, Yang J, et al. Afamilial cluster of pneumonia associated with the 2019 novel cor onavirus indicating person-to-person transmission: A study of a family cluster. The Lancet. 2020; 395(10223):514-23. [DOI:10.1016/S0140-6736(20)30154-9]

[3] Arefi MF, Poursadeqiyan M. A review of studies on the epidemic crisis of COVID-19 disease with a preventive approach. Work. 2020; 66(4):717-29. [DOI:10.3233/WOR-203218] [PMID] [DOI:10.3233/WOR-203218] [PMID]

[4] Wilder-Smith A, Freedman DO. Isolation, quarantine, social distancing and community containment: Pivotal role for old style public health measures in the novel coronavirus(2019- nCoV) outbreak. Journal of Travel Medicine. 2020; 27(2):taaa020.[DOI:10.1093/jtm/taaa020] [PMID] [PMCID]

[5] Bo HX, Li W, Yang Y, Wang Y, Zhang Q, Cheung T, et al. Post traumatic stress symptoms and attitude toward crisis mental health services among clinically stable patients with COVID-19 in China. Psychological Medicine. 2020:1-2. [DOI:10.1017/ S0033291720000999] [PMID] [PMCID]

[6] Sadati AK, Lankarani MHB, Bagheri Lankarani K. Risk society, global vulnerability and fragile resilience; Sociological view on the coronavirus outbreak. Shiraz E-Medical Journal. 2020; 21(4):e102263. [DOI:10.5812/semj.102263]

[7] Poursadeghiyan M, Abbasi M, Mehri A, Hami M, Raei M, Ebrahimi MH. Relationship between job stress and anxiety, depression and job satisfaction in nurses in Iran. The Social Sciences. 2016; 11(9):2349-55. https://acgih.ir/wp-content/ uploads/2018/09/989.pdf

[8] Yaribeygi H, Panahi Y, Sahraei H, Johnston TP, Sahebkar A. The impact of stress on body function: A review. EXCLI Journal. 2017; 16:1057-72. [DOI: 10.17179/excli2017-480]

[9] Huang C, Wang Y, Li X, Ren L, Zhao J, Hu Y, et al. Clinical features of patients infected with 2019 novel coronavirus in $\mathrm{Wu}$ han, China. Lancet (London, England). 2020; 395(10223):497506.[DOI:10.1016/S0140-6736(20)30183-5]

[10] Asadi N, Memarian R, Vanaki Z. Motivation to care: A qualitative study on Iranian nurses. The Journal of Nursing Research. 2019; 27(4):e34. [DOI:10.1097/jnr.0000000000000294] [PMID] [PMCID]

[11] Huang L, Rong Liu H. Emotional responses and coping strategies of nurses and nursing college students during COVID-19 outbreak. MedRxiv. 2020. https://www.medrxiv.org/ content/10.1101/2020.03.05.20031898v1

[12] Seccia R. The Nurse Rostering Problem in COVID-19 emergency scenario [Internet]. 2020 [Updated 2020 March 31]. Available from: http://www.optimization online.org/DB_ HTML/2020/03/7712.html

[13] Rahimian Boogar E, Nouri A, Oreizy H, Molavi H, Foroughi Mobarake A. [Relationship between adult attachment styles with job satisfaction and job stress in nurses (Persian)]. Iranian
Journal of Psychiatry and Clinical Psychology. 2007; 13(2):14857. http:/ /ijpcp.iums.ac.ir/article-1-165-en.html

[14] Nemati M, Ebrahimi B, Nemati F. Assessment of Iranian nurses' knowledge and anxiety toward COVID-19 during the current outbreak in Iran. Archives of Clinical Infectious Diseases. 2020; 15(COVID-19); e102848. [DOI:10.5812/archcid.102848]

[15] Soltani M, Khajei R, Rashidlamir A. Investigating the effect of aquatic aerobic training on the speed of walking in high and low-degree multiple sclerosis patients. Iranian Journal of Health and Physical Activity. 2011; 2(1):67. https://www.magiran.com/paper/837749

[16] Hosseinzadeh H, Mohammadi M, Shamshiri M. [The study of caring behaviors and its determinant factors from the perspective of nurses in educational hospitals of Ardabil(Persian)]. Journal of Health and Care. 2019; 21(3):203-11. [DOI:10.29252/ jhc.21.3.203]

[17] Soltani M, Hejazi SM, Noorian A, Zendedel A, Ashkanifar F. [The effect of aerobic training on the improvement of Expanded Disability Status Scale (EDSS) in multiple sclerosis patients (Persian)]. Journal of Medical Science of Islamic Azad University of Mashhad. 2009; 5(1):15-20. https://www.sid.ir/ En/Journal/ViewPaper.aspx?ID=204704.

[18] J Ehlers V. Is caring a lost art in nursing or is it a changing reality? Commentary on the editorial written by Juliet Corbin. International Journal of Nursing Studies. 2008; 45(5):802-4. [DOI:10.1016/j.ijnurstu.2007.09.004] [PMID]

[19] Lak Dizaji S, Rahmani A, Zamanzadeh V, Pashaei S. Perspec tives of patients and nurses on priorities of caring behaviors in critical care units: A comparative study. The Journal of Urmia Nursing and Midwifery Faculty. 2014; 12(9):866-73. http:// eprints.umsu.ac.ir/4135/

[20] Jackson D, Bradbury-Jones C, Baptiste D, Gelling L, Morin $\mathrm{K}$,Neville $\mathrm{S}$, et al. Life in the pandemic: Some reflections on nursing in the context of COVID-19. Journal of Clinical Nursing. 2020; 29(13-14):2041-3. [DOI:10.1111/jocn.15257] [PMID] [PMCID]

[21] Alipour A, Ghadami A, Alipour Z, Abdollahzadeh H. [Preliminary validation of the Corona Disease Anxiety Scale (CDAS) in the Iranian sample (Persian)]. Quarterly Journal of Health Psychology. 2020; 8(32):163-75. [DOI: 10.30473/ HPJ.2020.52023.4756]

[22] Watson R, Lea A. The Caring Dimensions Inventory(CDI): Content validity, reliability and scaling. Journal of $\mathrm{Ad}-$ vanced Nursing. 1997; 25(1):87-94. [DOI:10.1046/j.13652648.1997.1997025087.x][PMID]

[23] Salimi S, Azimpour A, Mohammadzadeh S, Fesharaki M. Psychometric properties of Persian version of the Caring DimensionInventory (PCDI-25). Iranian Journal of Nursing and Midwifery Research. 2014; 19(2):173-9. [PMCID] [PMID]

[24] Campbell AM. An increasing risk of family violence during the Covid-19 pandemic: Strengthening community collaborations to save lives. Forensic Science International: Reports. 2020; 2:100089. [DOI:10.1016/j.fsir.2020.100089] [PMCID]

[25] Pappa S, Ntella V, Giannakas T, Giannakoulis VG, Papoutsi E, Katsaounou P. Prevalence of depression, anxiety, and insomnia among healthcare workers during the COVID-19 pandemic: A systematic review and meta-analysis. Brain, Behavior, and Immunity. 2020; 88:901-7. [DOI:10.2139/ssrn.3594632] 
[26] Xuehua L, Li M, Fangiang ME. Psychological stress of nurses in SARS wards. Chinese Mental Health Journal. 2003; 17:526-7. https://en.cnki.com.cn/Article_en/CJFDTotal-ZXWS200308003.htm

[27] Khalilzadeh Naghneh MH, Tafreshi MZ, Naderi M, Shakeri N, Bolourchifard F, Goyaghaj NS. The relationship between organi zational commitment and nursing care behavior. Electronic Physician. 2017; 9(7):4835-40. [DOI:10.19082/4835] [PMID] [PMCID]

[28] Rasti F, Joolaee S, Ghiyasvandian S, Haghan H. [Patients' perceptions of caring behaviors in oncology settings (Persian)]. Iranian Journal of Nursing Research. 2014; 9(1):59-67. http:/ /ijnr.ir/ browse.php?a_id=1335\&sid=1\&slc_lang=en

[29] Kaveh M, Davari-tanha F, Varaei S, Shirali E, Shokouhi N, Nazemi $P$, et al. Anxiety levels among Iranian health care workersduring the COVID-19 surge: A cross-sectional study. MedRxiv. 2020. [DOI:10.1101/2020.05.02.20089045]

[30] Mehrabi T, Parvin N, Yazdani M, Rafat NA. A study of the severity of some occupational stresses in nurses. Iranian Journal of Nursing and Midwifery Research. 2008; 12(1):21-4. http://ijnmr. mui.ac.ir/index.php/ijnmr/article/view/6/6

[31] Mohebbifar R, Kiaei MZ, Khosravizadeh O, Sadeghi T,Ahansazan $\mathrm{H}$. [Job stress and its related factors in nurses of Qaz vin University of Medical Sciences (Persian)]. The Journal of Medical Education and Development. 2015; 7(1):55-63. http:/ / eprints. qums.ac.ir/2779/

[32] Rodrigues VM, Ferreira AS. Stressors in nurses working in Intensive Care Units. Revista latino-americana de enfermagem. 2011； 19(4):1025-32. [DOI:10.1590/S0104-11692011000400023] [PMID]

[33] Cho I, Ahn S, Kim SY, Park YS, Kim HW, Lee SO, et al. Depression of married and employed women based on social-roletheory. Journal of Korean Academy of Nursing. 2012; 42(4):496-507. [DOI:10.4040/jkan.2012.42.4.496] [PMID]

[34] Al Thobaity A, Alshammari F. Nurses on the frontline against the COVID-19 Pandemic: An integrative review. Dubai Medical Journal. 2020; 3(3):87-92. [DOI:10.1159/000509361] [PMCID]

[35] Derakhshanpour F, Izadyar H, Shahini N, Vakili MA. [Anxiety levels in the primary school students in Gorgan (Persian)]. Pajoohandeh. 2016; 21(1):30-4. http://pajoohande.sbmu.ac.ir/ article-1-2137-en.html 